\title{
0246 HOW TO PREVENT DOG BITE INJURIES? CHILDREN MISINTERPRET DOGS FACIAL EXPRESSIONS
}

K Meints*, A Racca, N Hickey Correspondence: University of Lincoln, School of Psychology, Brayford Pool, Lincoln LN6 7TS, UK

10.1136/ip.2010.029215.246

Almost half of school children reported that they were bitten (Beck \& Jones,1985; Spiegel, 2000) and in other research, 20\% of dog-owning parents reported their child bitten (Wilson, Dwyer and Bennett, 2003, see also Lakestani, Donaldson, Verga \& Waran, 2006). Child-initiated interactions with the dog trigger up to $86 \%$ of injuries at home. Recently, it was found that young children do not discriminate a dogs body signals, but look mainly at the dogs face (Lakestani et al., 2006). While there has been anecdotal evidence that children mistake an angry, teeth-baring dog face for a smiling one, there is to date no systematic empirical evidence on the misinterpretation of dogs facial expressions. We tested 4-, 5-, 6-year-old children and adults on neutral, aggressive and happy human and dog facial expressions. Results show that while adults make hardly any mistakes (less than $1 \%$ ) on both stimulus types (dog and human faces), 69\% of 4-year-olds interpreted aggressive dog faces as smiling and happy. Five-year-olds show $35 \%$ and 6 -year-olds $25 \%$ misinterpretations whereas they are over $90 \%$ correct on all human facial expressions. This result indicates a severe lack in interpretation abilities in children of facial expressions of dogs which could contribute to the high incidence of dog bites, especially in younger children. Given this result, we can advise children and parents to prevent injuries and inform dog bite prevention programmes to help prevent further dog bite incidents. 